\title{
Minimum Critical Masses at the Portsmouth Gaseous Diffusion Plant
}

\author{
June 1994
}

by

Robert W. Tayloe, Jr.

Battelle

Thomas C. Davis

Private Consultant to Battelle

Under Contract 400183

to

MARTIN MARIETTA UTILITY SERVICES, INC.

PORTSMOUTH GASEOUS DIFFUSION PLANT

P.o. Box 628 Piketon, Ohio 45661

Under Contract USECHO-93-C-0001

to the

U.S. ENRICHMENT CORPORATION 


\section{DISCLAIMER}

This report was prepared as an account of work sponsored by an agency of the United States Government. Neither the United States Government nor any agency thereof, nor any of their employees, make any warranty, express or implied, or assumes any legal liability or responsibility for the accuracy, completeness, or usefulness of any information, apparatus, product, or process disclosed, or represents that its use would not infringe privately owned rights. Reference herein to any specific commercial product, process, or service by trade name, trademark, manufacturer, or otherwise does not necessarily constitute or imply its endorsement, recommendation, or favoring by the United States Government or any agency thereof. The views and opinions of authors expressed herein do not necessarily state or reflect those of the United States Government or any agency thereof. 


\section{DISCLAIMER}

\section{Portions of this document may be illegible in electronic image products. Images are produced from the best available original document.}




\section{DISTRIBUTION}

\section{MARTIN MARIETTA UTILITY SERVICES, INC.}
R. Newvahner
J. Harris
C. Haught
R. Oxenham
R. Lemming

D. D'Aquila (10)

Central Files (2)

X-710 Technical Library (2)

Technical Review (2)

\section{PADUCAH}
C. Dean (2)
S. Wachtel
D. Baltimore
V. Risner
S. Negron
R. Tayloe
W. Simmons
R. Denning
B. Lee
T. Davis
D. Lindenschmidt
C. Dobelbower 


\section{TABLE OF CONTENTS}

Page

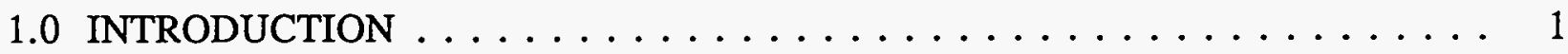

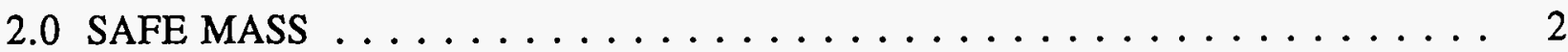

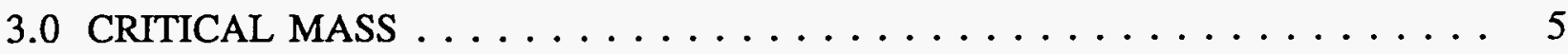

4.0 COMPARISON TO OTHER DATA . . . . . . . . . . . . 7

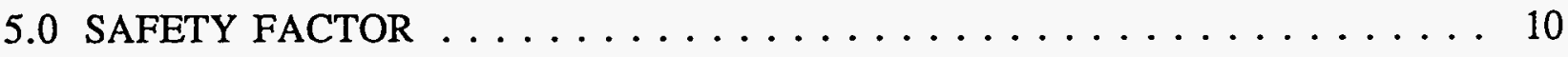

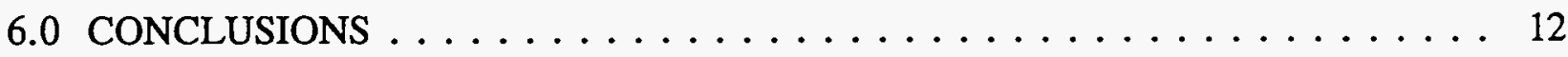

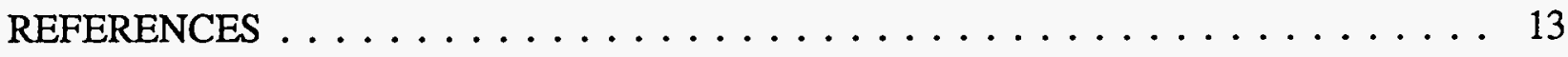

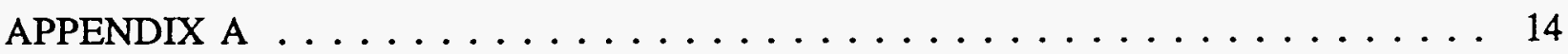

\section{LIST OF TABLES}

Table 1. Data Used for Safe-Mass Curve Fit ................... 3

Table 2. Data Used for Critical-Mass Curve Fit ................. 5

Table 3. Data Points Used for Comparison . . . . . . . . . . . . . . . . 8

Table A-1. Calculated Safe and Critical Masses . . . . . . . . . . . . 15

\section{LIST OF FIGURES}

Figure 1. Safe Mass Fit to GAT-225 Data . . . . . . . . . . . . . . . . . . . . 4

Figure 2. Critical Mass Fit to ORNL/TM-12292 Data . . . . . . . . . . . . . 6

Figure 3. Comparison to Data Tabulated Safe and Critical Masses ... . . . . . . 9

Figure 4. Safety Factor Ratio of Critical to Safe Mass . . . . . . . . . . . . . 11 


\subsection{INTRODUCTION}

This report presents a tabulation of safe masses and minimum critical masses for uranium $(\mathrm{U})$. These minimum critical mass and safe mass tables were obtained by interpolating between the values reported in the literature to obtain values as a function of enrichment within the 1.5 percent to 100 percent range. Equivalent mass values for uranium-235 $\left(\mathrm{U}^{235}\right)$, uranium hexafluoride $\left(\mathrm{UF}_{6}\right)$, and uranyl fluoride $\left(\mathrm{UO}_{2} \mathrm{~F}_{2}\right)$ have been generated from the safe mass and minimum critical masses for uranium. 


\subsection{SAFE MASS}

The safe mass tables were derived from the safe mass values historically used at the Portsmouth Gaseous Diffusion Plant, which are delineated in GAT-225 (Reference 1). These data points, which are shown in Table 1, were used to develop an equation for safe mass of $\mathrm{U}^{235}$ as a function of enrichment over the range of 1.5 to 93 percent.

The curve that results from the equation is shown in Figure 1. The safe mass value at 93 percent is 350 grams, which is equal to the safe mass limit given in the GAT-225 for 100 percent enriched uranium. Consequently, as shown in Figure 1, the safe mass in the of range 93 to 100 percent enrichment is assumed to be a constant value of 350 grams. Using the safe masses of $U^{235}$ for each enrichment, the safe masses of uranium were calculated based on the relative atomic weights. The calculations assume the uranium consisted entirely of $U^{235}$ and $U^{238}$ (i.e., the percentage of $U^{234}$ was assumed to be negligible). The safe masses of $\mathrm{UF}_{6}$ and $\mathrm{UO}_{2} \mathrm{~F}_{2}$ were then calculated based on their relative molecular weights. Detailed results for uranium, $\mathrm{U}^{235}, \mathrm{UF}_{6}$, and $\mathrm{UO}_{2} \mathrm{~F}_{2}$ are shown in Appendix A. 
Table 1. Data Used for Safe-Mass Curve Fit

\begin{tabular}{|c|c|}
\hline $\begin{array}{c}\mathrm{U}^{235} \\
(\mathrm{wt} \%)\end{array}$ & $\begin{array}{c}\text { Safe Mass (grams) } \\
\text { Reference: GAT-225, Table I }\end{array}$ \\
\hline 1.5 & 4500 \\
\hline 1.75 & 3150 \\
\hline 2 & 2400 \\
\hline 2.5 & 1500 \\
\hline 3 & 1180 \\
\hline 3.5 & 1000 \\
\hline 4 & 900 \\
\hline 5 & 800 \\
\hline 6 & 740 \\
\hline 8 & 650 \\
\hline 10 & 600 \\
\hline 12 & 560 \\
\hline 15 & 520 \\
\hline 20 & 480 \\
\hline 30 & 440 \\
\hline 40 & 410 \\
\hline 50 & 390 \\
\hline 75 & 360 \\
\hline 93 & 350 \\
\hline
\end{tabular}


POEF-SH-20

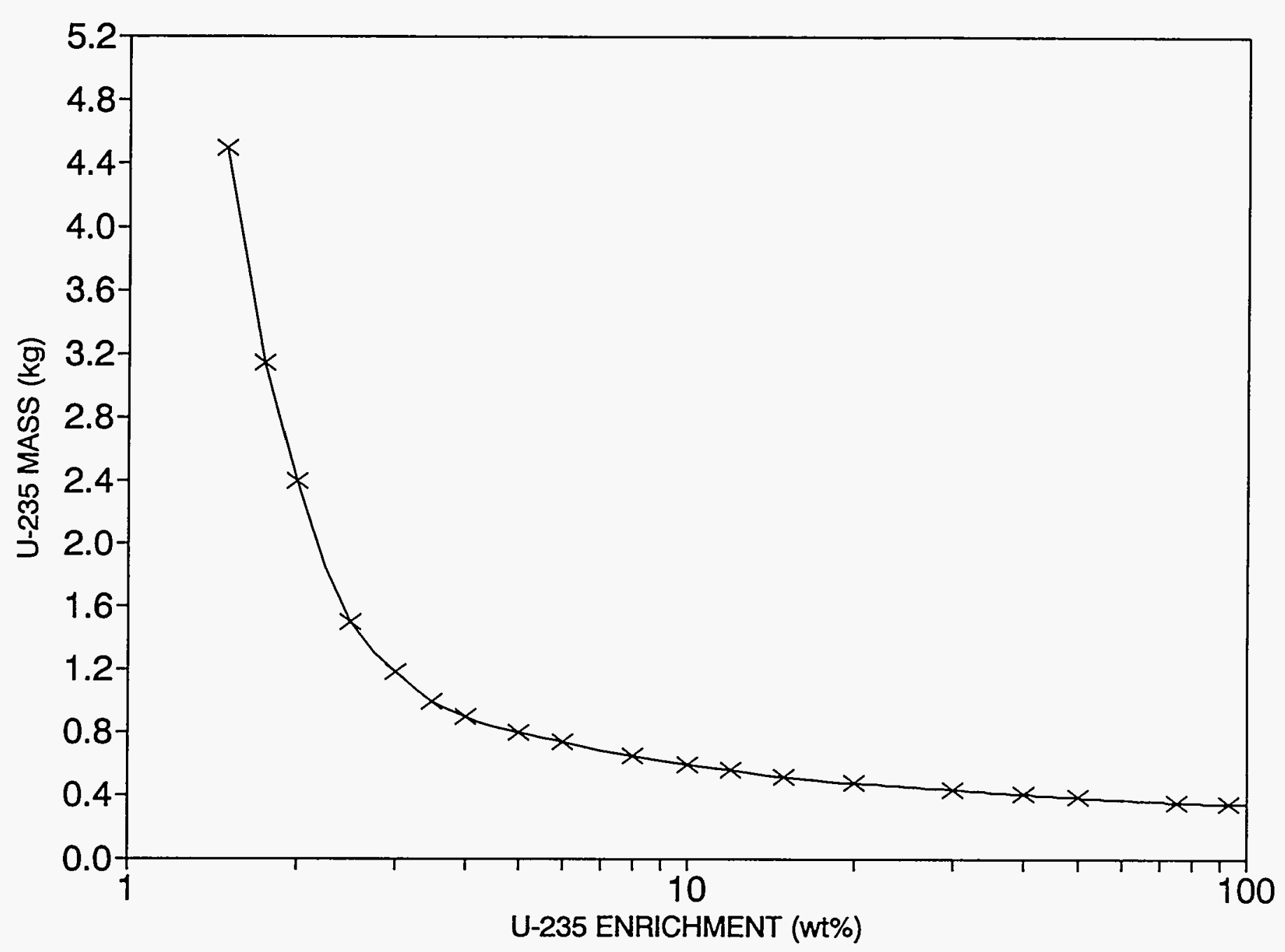

Figure 1. Safe Mass

Fit to GAT-225 Data

Final Report

June 1994 


\subsection{CRITICAL MASS}

The data used to develop the curve-fit equation for critical masses were derived from Table B.2 of ORNL/TM-12292 (Reference 2). These data represent the critical mass at an $\mathrm{H} / \mathrm{X}$ of 500 for uranyl fluoride-water (UO2F2-H2O) systems in fully-water-reflected spherical geometry determined using the SCALE 4.0 package. GAT-225 specifies an H/X of 500 if mass is the parameter of nuclear safety control. ORNL/TM-12292 indicates that an $\mathrm{H} / \mathrm{X}$ of 500 results in a near-minimum predicted critical mass. These data points, which are shown in Table 2, were used to develop an equation for critical mass of $U^{235}$ as a function of enrichment over the range of 1.5 to 100 percent. The lowest enrichment used to determine the curve was 1.4 percent. However, for consistency with safe mass data, Appendix A presents results for enrichments of 1.5 percent and higher. The curve that results from the equation is shown in Figure 2.

Table 2. Data Used for Critical-Mass Curve Fit

\begin{tabular}{|c|c|}
\hline $\begin{array}{c}U^{235} \\
(w i)\end{array}$ & $\begin{array}{c}\left.\text { Critical Mass (grams } U^{235}\right) \\
\text { Reference: ORNL/TM-12292, Table B.2 }\end{array}$ \\
\hline 1.4 & 23773 \\
\hline 1.7 & 9881 \\
\hline 2 & 6264 \\
\hline 3 & 3077 \\
\hline 4 & 2221 \\
\hline 5 & 1851 \\
\hline 7 & 1505 \\
\hline 10 & 1275 \\
\hline 20 & 1021 \\
\hline 50 & 852 \\
\hline 10 & 775 \\
\hline
\end{tabular}


POEF-SH-2O

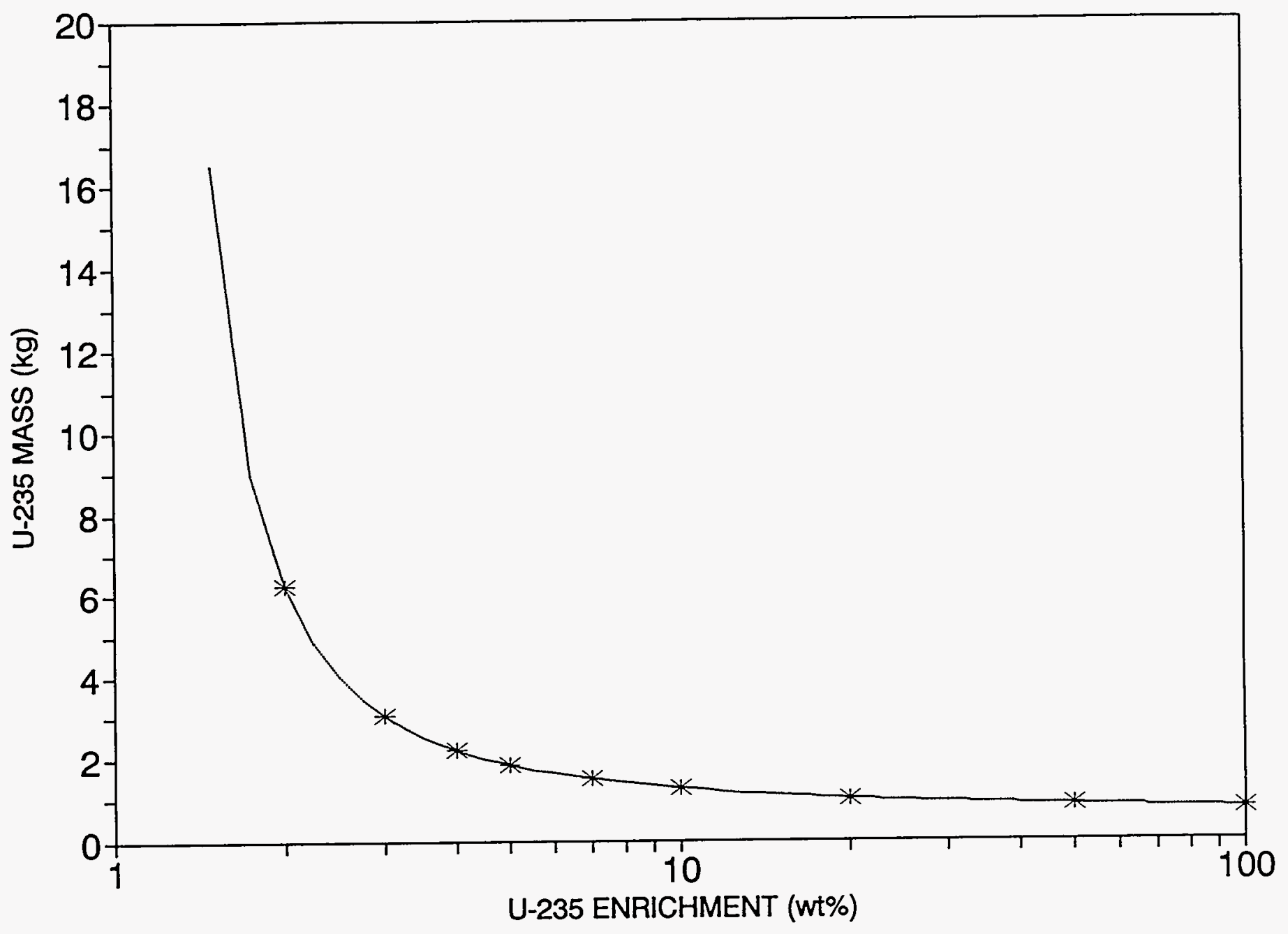

Figure 2. Critical Mass

Fit to ORNL/TM-12292 Data 


\subsection{COMPARISON TO OTHER DATA}

The resulting safe and critical mass curves were compared to available data from ANSI/ANS-8.1, LA-10860, and ARH-600 (References 3, 4, and 5). The sources of the data points are given in Table 3, and the data points are included in Figure 3.

Figure 3 shows that the calculated safe values are conservative with respect to the other data over the full range of enrichments. The estimated critical masses are also conservative with respect to the data for homogeneous critical systems from LA-10860 and ARH-600 for enrichments above 5 percent.

At enrichments less than or equal to 5 percent, the LA-10860 data points for homogeneous critical system, (which are estimated from Figure 22 of LA-10860) are slightly less that the values derived from the ORNL/TM-12292 calculated data. The LA-10860 data point for low-enriched heterogeneous critical systems (see Figure 22 of LA-10860) are less than that predicted for a homogeneous system. The subcritical limits for aqueous solutions of $\mathrm{UO}_{2} \mathrm{~F}_{2}$ from ANSI/ANS-8.1 (see Tables 1 and 6 of ANSI/ANS-8.1) are less than the estimate of the critical mass determined by the curve fit between 2 and 5 percent. The ANSI/ANS-8.1 data represent a subcritical limit and were determined considering the calculational uncertainties.

Overall, the tabulated values are comparable to the data presented in other references at enrichments above 5 percent. There are, however, significant differences between the estimated critical masses reported in the various sources at low enrichments. The differences reflect calculational uncertainties and different modeling assumptions.

\section{Final Report}

June 1994 
Table 3. Data Points Used for Comparison

\begin{tabular}{|c|c|c|}
\hline $\begin{array}{l}U^{235} \\
(w t \%)\end{array}$ & $\begin{array}{l}\text { Critical } \\
\text { Mass U } \mathbf{U}^{235} \\
(\mathrm{~kg})\end{array}$ & Reference \\
\hline 2 & 3.2 & $\begin{array}{l}\text { LA-10860, Figure } 22 \text {, Heterogeneous water-reflected data } \\
\text { point, minimum critical mass }\end{array}$ \\
\hline 2 & 5.2 & $\begin{array}{l}\text { LA-10860, Figure } 22 \text {, Homogeneous water- reflected data } \\
\text { point, minimum critical mass }\end{array}$ \\
\hline 3 & 2.2 & $\begin{array}{l}\text { LA-10860, Figure } 22 \text {, Heterogeneous water-reflected data } \\
\text { point, minimum critical mass }\end{array}$ \\
\hline 3 & 2.6 & $\begin{array}{l}\text { LA-10860, Figure } 22 \text {, Homogeneous water- reflected data } \\
\text { point, minimum critical mass }\end{array}$ \\
\hline 5 & 1.6 & $\begin{array}{l}\text { LA-10860, Figure } 22 \text {, Heterogeneous water-reflected data } \\
\text { point, minimum critical mass }\end{array}$ \\
\hline 5 & 1.8 & $\begin{array}{l}\text { LA-10860, Figure } 22 \text {, Homogeneous water- reflected data } \\
\text { point, minimum critical mass }\end{array}$ \\
\hline 15 & 1.15 & $\begin{array}{l}\text { LA-10860, Figure } 22 \text {, Homogeneous water- reflected data } \\
\text { point, minimum critical mass }\end{array}$ \\
\hline 40 & 1.00 & $\begin{array}{l}\text { LA-10860, Figure } 22, \text { Homogeneous water- reflected data } \\
\text { point, minimum critical mass }\end{array}$ \\
\hline 93 & 0.814 & $\begin{array}{l}\text { LA-10860, Figure } 22 \text {, Homogeneous water- reflected data } \\
\text { point, minimum critical mass }\end{array}$ \\
\hline 100 & 0.806 & ARH-600, Figure III.B.6(100)-2, minimum critical mass \\
\hline 2 & 8.00 & $\begin{array}{l}\text { ANSI/ANS-8.1, Table 6, subcritical limit for aqueous solutions } \\
\text { of } \mathrm{UO}_{2} \mathrm{~F}_{2}\end{array}$ \\
\hline 3 & 2.75 & $\begin{array}{l}\text { ANSI/ANS-8.1, Table 6, subcritical limit for aqueous solutions } \\
\text { of } \mathrm{UO}_{2} \mathrm{~F}_{2}\end{array}$ \\
\hline 4 & 1.98 & $\begin{array}{l}\text { ANSI/ANS-8.1, Table 6, subcritical limit for aqueous solutions } \\
\text { of } \mathrm{UO}_{2} \mathrm{~F}_{2}\end{array}$ \\
\hline 5 & 1.64 & $\begin{array}{l}\text { ANSI/ANS-8.1, Table 6, subcritical limit for aqueous solutions } \\
\text { of } \mathrm{UO}_{2} \mathrm{~F}_{2}\end{array}$ \\
\hline 10 & 1.07 & $\begin{array}{l}\text { ANSI/ANS-8.1, Table 6, subcritical limit for aqueous solutions } \\
\text { of } \mathrm{UO}_{2} \mathrm{~F}_{2}\end{array}$ \\
\hline 100 & 0.76 & $\begin{array}{l}\text { ANSI/ANS-8.1, Table 1, subcritical limit for aqueous solutions } \\
\text { of } \mathrm{UO}_{2} \mathrm{~F}_{2}\end{array}$ \\
\hline
\end{tabular}




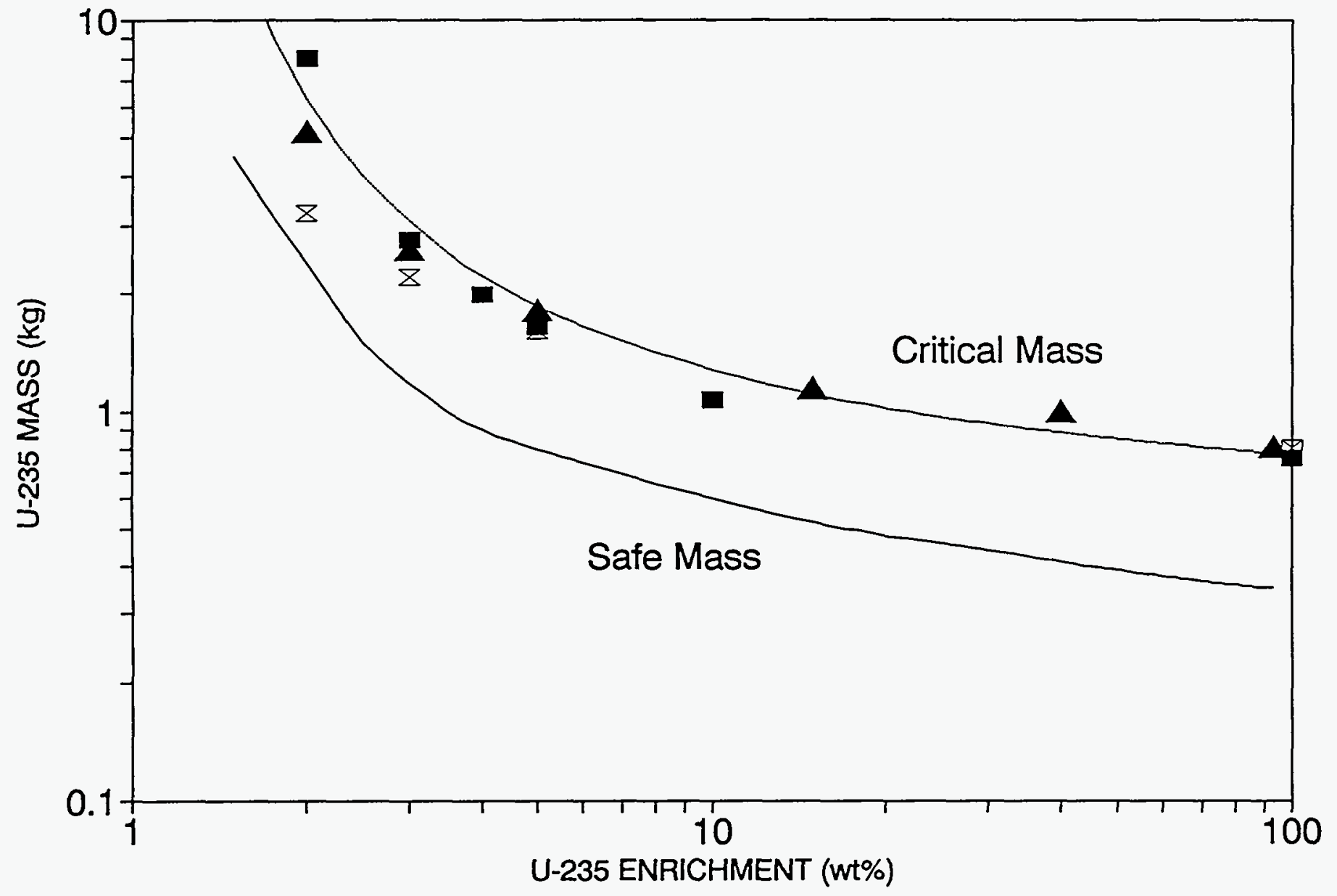




\subsection{SAFETY FACTOR}

Historically, the gaseous diffusion plants have incorporated a factor of safety with minimum critical masses to generate "safe" masses. In order to account for errors in double batching or to reduce the potential for criticality as a result from errors in conversion from metric mass units (kilograms) to more conventional units of weight (pounds), the safe mass was taken as 43.5 percent of the critical value. This percentage equates to a safety factor of 2.3 .

Figure 4 presents the calculated safety factor over the enrichment range of interest, 1.5 to 100 percent. The safety factor is calculated as the ratio of the estimated critical mass to the estimated safe mass at each enrichment. Figure 4 shows that the safety factors vary from 2.1 to 2.3 for enrichments in the range of 5 to 100 percent. These values are comparable to the safety factor of 2.3 that was used to develop the safe masses in GAT-225. However, the safety factors are somewhat larger at lower enrichments (as much as 3.7 at 1.5 percent enrichment). 


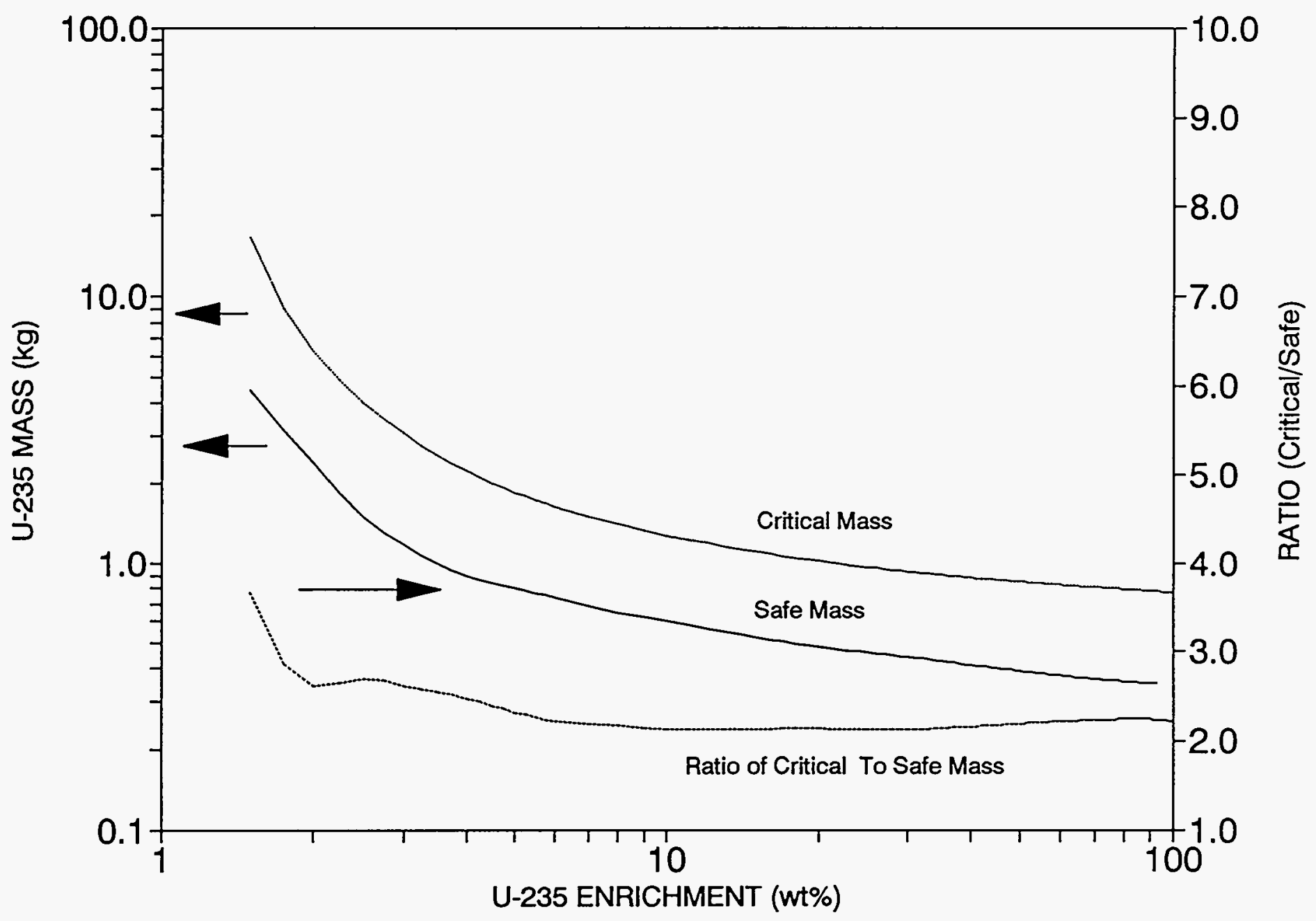

Figure 4. Safety Factor

Ratio of Critical to Safe Mass 


\subsection{CONCLUSIONS}

The safe mass values are an interpolation of the GAT-225 safe mass parameters. The tabulated values can be used over the full range of enrichment for criticality safety analysis calculations.

The tabulated critical mass values represent an estimate of the mass required for a system that is exactly critical with the assumptions of an $\mathrm{H} / \mathrm{X}$ of 500 and fully-waterreflected spherical geometry. The tabulated values are based on recent calculations using the SCALE package. The tabulated values are comparable to the data presented in other references at enrichments above 5 percent. There are, however, significant differences between the values for critical masses reported in the literature at lower enrichments.

The tabulated critical mass values do not incorporate a safety factor and do not represent a conservative estimate of the minimum critical mass. The tabulated critical masses are often useful in performing risk assessments. However, all criticality safety analyses should incorporate a safety factor, and limiting conditions should be based on safe parameters. 


\section{REFERENCES}

1. GAT-225, Revision 4, Feuerbacher, J.L., "Nuclear Criticality Safety Guide for the Portsmouth Gaseous Diffusion Plant," Portsmouth Gaseous Diffusion Plant, March 1981.

2. ORNL/TM-12292, Jordan, W.C., Turner, J.C., "Estimated Critical Conditions for $\mathrm{UO}_{2} \mathrm{~F}_{2}-\mathrm{H}_{2} \mathrm{O}$ Systems in Fully Water-Reflected Spherical Geometry", Oak Ridge National Laboratory, December 1992.

3. ANSI/ANS-8.1, "Nuclear Criticality Safety in Operations with Fissionable Materials Outside Reactors", American Nuclear Society, 1983.

4. LA-10860-MS, Paxton, H.C., and Pruvost, N.L., "Critical Dimensions of Systems Containing U ${ }^{235}, \mathrm{Pu}-239$, and U-233 - 1986 Revision", Los Alamos National Laboratory, July 1987.

5. ARH-600, Carter, R.D., et al, "Criticality Handbook", volume II, Atlantic Richfield Hanford Company, March 1976.

6. K-1019, Revision 5, Henry, H.F., et al, "Criticality Data and Nuclear Safety Guide Applicable to The Oak Ridge Gaseous Diffusion Plant", Oak Ridge Gaseous Diffusion Plant, May 1959. 


\section{APPENDIX A}

CALCULATED RESULTS

SAFE AND CRITICAL MASSES

FOR U ${ }^{235}$, URANIUM,

$\mathrm{UF}_{6}, \mathrm{AND} \mathrm{UO}_{2} \mathrm{~F}_{2}$ 
Iupld uo!snfl!a snoasng ymousuod

$\varsigma_{I} 28 n_{d}$

ayt to um! upג Lof sasspW

$\$ 66 I$ aun $\Gamma$

jJodəy Ieutet

\begin{tabular}{|c|c|c|c|c|c|c|c|c|}
\hline $050^{\circ} \mathrm{L}$ & $850^{\circ} 8$ & ऽかザ & $5 \Sigma 0^{\circ} l$ & ElE. & $\angle 8 L^{\circ} \varepsilon$ & $655^{\circ} 2$ & $987^{\circ} 0$ & 61 \\
\hline $875: 2$ & $829^{\circ} 8$ & $0 £ 8^{\circ} 5$ & $670^{\circ} 1$ & $\angle 7 S^{\circ} \varepsilon$ & $550^{-7}$ & $07 L^{\circ} 2$ & $\varepsilon 67^{\circ} 0$ & 81 \\
\hline $611 \cdot 8$ & $082^{\circ} 6$ & $1 \angle 2^{\circ} 9$ & $990^{\circ} 1$ & $918^{\circ} \varepsilon$ & 29ع־ท & $\angle 76^{\circ} 2$ & $105^{\circ} 0$ & $\angle L$ \\
\hline $9 L L^{\prime} 8$ & $\angle E 0^{\circ} O L$ & $62 L^{\circ} 9$ & $580^{\circ} l$ & $921^{\circ} 7$ & $\left.\angle L L^{\circ}\right\rangle$ & $\angle 8 L^{\circ} \varepsilon$ & OLS"0 & 91 \\
\hline $275^{\circ} 6$ & $206^{\circ} 01$ & $\angle L E^{*} L$ & $901^{\circ} L$ & $887^{\circ} 7$ & $0 \Sigma l^{\circ} \varsigma$ & $\angle 97^{\circ} \varepsilon$ & $025^{\circ} 0$ & Sl \\
\hline St7.0l & $856^{\circ} \mathrm{Ll}$ & $890^{\circ} 8$ & $0 \varepsilon l^{\circ} l$ & $\varepsilon 16^{\circ} 7$ & $919^{\circ} \mathrm{S}$ & $S 6 L^{\circ} \varepsilon$ & LES"0 & 71 \\
\hline $725 \cdot 11$ & $2 L l^{\circ} \varepsilon l$ & $206^{\circ} 8$ & $\angle S L^{\circ} l$ & 127.5 & $261^{\circ} 9$ & $881^{\circ} 7$ & $7 \pitchfork 5^{\circ} 0$ & $\varepsilon$ \\
\hline$\overline{\Sigma S 8^{\circ} \mathrm{ZL}}$ & $899^{\circ} 71$ & 716.6 & $06 l^{\circ} !$ & $170^{\circ} 9$ & $506^{\circ} 9$ & $\angle 99^{\circ} \eta$ & $095^{\circ} 0$ & 21 \\
\hline $757^{\circ} 71$ & $025^{\circ} 91$ & $99 ! \cdot 1 l$ & $822^{\circ} !$ & $118^{\circ} 9$ & $58 L^{\circ} L$ & $292^{\circ} \mathrm{S}$ & $6 \angle 5^{\circ} 0$ & 11 \\
\hline $705^{\circ} 91$ & $\varepsilon 98^{\circ} 81$ & $0 S L^{\circ} 2 l$ & $S \angle Z^{*} b$ & $99 L^{\circ} L$ & $\angle \angle 8^{\circ} 8$ & $000^{\circ} 9$ & $009^{\circ} 0$ & 01 \\
\hline $0 \angle l^{\circ} 61$ & $116^{\circ} 12$ & $118^{\circ} \mapsto l$ & 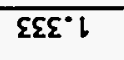 & $\varepsilon 56^{\circ} 8$ & $\varepsilon \varepsilon Z^{*} 01$ & $\angle 16^{\circ} 9$ & $\varepsilon 29^{\circ} 0$ & 6 \\
\hline$\varepsilon 9 L^{\circ} Z 2$ & $\angle 10^{\circ} 92$ & $285^{\circ} \angle 1$ & $\angle 07^{\circ} l$ & $915^{\circ} 01$ & $020^{\circ} \mathrm{Zl}$ & $52 L^{\circ} 8$ & $059^{\circ} 0$ & 8 \\
\hline$\angle 28^{\circ} \angle 2$ & $708^{\circ}$ IE & $005^{\circ} 12$ & $\operatorname{SOS}^{\circ} 1$ & $Z \eta L^{\circ} Z L$ & E95“クl & $5+8^{\circ} 6$ & $689^{\circ} 0$ & $L$ \\
\hline $027^{\circ} \varsigma \varepsilon$ & $287^{\circ} 07$ & $\angle 9 E^{\circ} \angle 2$ & $249^{\circ} !$ & $\varepsilon 96^{\circ} \mathrm{Sl}$ & 772.81 & $\varepsilon \varepsilon \varepsilon^{\bullet} Z b$ & $04 L^{\circ} 0$ & 9 \\
\hline $076^{\circ} \angle \varepsilon$ & $29 \varepsilon^{\circ} \Sigma \eta$ & $71 \varepsilon^{\circ} 62$ & $989^{\circ} 1$ & $896^{\circ} 91$ & $26 \varepsilon^{\circ} 6 \mathrm{l}$ & $0 l^{\circ} \varepsilon b$ & $7 S L^{\circ} 0$ & $S L^{\circ} S$ \\
\hline $608^{\circ} 07$ & $179^{\circ} 97$ & IES IE & $7 \varepsilon L^{\circ} l$ & $9 \angle 0^{\circ} 81$ & $659^{\circ} 02$ & $996^{\circ} \mathrm{EL}$ & $89 L^{\circ} 0$ & $5^{\circ} 5$ \\
\hline ட0ட・カウ & $507^{\circ} 0 S$ & $\varsigma \angle 0^{\circ} 7 \varepsilon$ & $682^{\circ} 1$ & $2 l \varepsilon^{\circ} 6 l$ & $1 \angle 0^{\circ} \mathrm{ZZ}$ & $126^{\circ} 71$ & $\varepsilon 8 L^{\circ} 0$ & $\$ 2 \cdot 5$ \\
\hline $216^{\circ} \angle 7$ & $8 S L^{\circ} 75$ & $020^{\circ} \angle \varepsilon$ & $158^{\circ} \mathrm{L}$ & $80 L^{\circ} 02$ & $999^{\circ} \mathrm{EZ}$ & $000^{\circ} 91$ & $008^{\circ} 0$ & 5 \\
\hline $0 \angle \varepsilon^{\circ} 25$ & $558^{\circ} 65$ & $597^{\circ} 07$ & $226^{\circ} 1$ & $80 \varepsilon^{\circ} 22$ & $967^{\circ} \mathrm{SL}$ & $\angle E Z^{\circ} \angle L$ & $618^{\circ} 0$ & $S L^{\circ}$ \\
\hline$\angle S 9^{\circ} \angle S$ & $\$ 68^{\circ} \$ 9$ & OSS"サ & $500^{\circ} 2$ & $U l^{-\curvearrowleft \eta 2}$ & $L \varepsilon 9^{\circ} L Z$ & $189^{\circ} 81$ & $178^{\circ} 0$ & Sי7 \\
\hline $050^{\circ} 79$ & $6 \angle L^{\circ} \varepsilon L$ & SL7・67 & $201^{\circ} \mathrm{Z}$ & $507^{\circ} 92$ & $S \angle L^{\circ} 0 \mathcal{L}$ & $107^{\circ} 02$ & $\angle 98^{\circ} 0$ & Sट"H \\
\hline $098^{\circ} \mathrm{KL}$ & $\angle 21 \cdot 28$ & S2S. SS & $122 \cdot 2$ & $611 \cdot 62$ & $082^{\circ} \varepsilon \varepsilon$ & $005^{\circ} 22$ & $006^{\circ} 0$ & 7 \\
\hline $6<9^{\circ} 18$ & $87 \varepsilon^{\circ} \varepsilon 6$ & 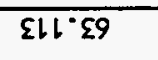 & $29 \varepsilon^{\circ} 2$ & $625^{\circ} 2 \varepsilon$ & $\angle L^{\circ} \angle \varepsilon$ & SEl ${ }^{\circ} \varsigma 2$ & $\varepsilon 76^{\circ} 0$ & $\overline{S L} \cdot \bar{\varepsilon}$ \\
\hline เクン・ク6 & $70 L^{\circ} \angle O L$ & $618^{\circ} 21$ & $675^{\circ} 2$ & $9 \angle 6^{\circ} 9 \varepsilon$ & $652^{\circ} 27$ & $125^{\circ} 82$ & $000^{\circ} 1$ & $\zeta^{\circ} \varepsilon$ \\
\hline $2 \angle 9^{\circ} 01 L$ & $\varepsilon 87^{\circ} 921$ & $\angle 15^{\circ} 58$ & $6 L 2 \cdot 2$ & $\varepsilon 96^{\circ} 27$ & $101 \cdot 67$ & $861^{\circ}$ EE & $620^{\circ} \mathrm{L}$ & $\varsigma Z^{*} \boldsymbol{\varepsilon}$ \\
\hline $9 \varepsilon L^{\circ} 2 \varepsilon L$ & $669^{\circ}$ LSL & $\angle 95^{\circ} 201$ & $\angle 0^{\circ} \varepsilon$ & $\varepsilon 06^{\circ} 05$ & $S L L^{\circ} 8 S$ & EEE $6 \varepsilon$ & $081 \cdot l$ & $\varepsilon$ \\
\hline$\varepsilon\llcorner\overbrace{} \varepsilon 9 l$ & $8 S L^{\circ} 981$ & $2 \angle 2^{\circ} 921$ & $2 \angle 7^{\circ} \varepsilon$ & $677 * 19$ & $822^{\circ} 02$ & $\varepsilon 87^{\circ} \angle 7$ & $90 \varepsilon^{\circ} l$ & $\Phi L^{\prime \prime 2}$ \\
\hline $5 \Sigma 5^{\circ} 802$ & SZE $8 \varepsilon 2$ & $6 \varepsilon l^{\circ}\lfloor 9 l$ & $820^{\circ} 7$ & $849^{\circ} \mathrm{LL}$ & $07 L^{\circ} 88$ & $000^{\circ} 09$ & $005^{\circ} l$ & $s^{*} 2$ \\
\hline $060^{\circ} 082$ & $00 l^{\circ} 0 Z \varepsilon$ & $2 \varepsilon 7^{\circ} 9 l 2$ & $0 \angle 8^{\circ} 7$ & L77゚90L & $\$ 59^{\circ} 121$ & $752^{\circ} 28$ & $158^{\circ} !$ & $52 \cdot 2$ \\
\hline SIE. SO† & $\varepsilon L C^{*} \varepsilon 97$ & $002^{\circ} \varepsilon l \varepsilon$ & $492^{\circ} 9$ & $\varepsilon 62^{\circ} \leqq S L$ & $9 \angle \eta^{\circ} \measuredangle L l$ & $000^{\circ} 021$ & $007^{\circ} 2$ & 2 \\
\hline $911 \cdot+99$ & $086^{\circ} 85 L$ & $\angle 8 L^{\circ} \varepsilon I S$ & $186^{\circ} 8$ & $8 \varepsilon 6^{\circ} 2 \varepsilon 2$ & $212^{\circ} 992$ & $000^{\circ} 081$ & $0 S L^{\circ} \varepsilon$ & $S L^{\circ} l$ \\
\hline$\angle Z Z^{*} \gg Z \gg L$ & $\Sigma 99^{\circ} \angle 291$ & $195^{\circ} 0016$ & $805^{\circ} 91$ & $822^{\circ} 88 \varepsilon$ & $289^{\circ} \varepsilon \not \dagger$ & $000^{\circ} 00 \Sigma$ & $005^{\circ} 7$ & $s^{\bullet} l$ \\
\hline$z_{d} z_{0}$ n & ${ }^{9} \mathrm{An}$ & untuedn & $9 \notin z^{n}$ & $z_{y^{2} \text { on }}$ & ${ }^{8} \mathrm{An}$ & un!tuesn & $9 \varepsilon 2^{n}$ & \multirow{2}{*}{$\begin{array}{l}(\%, \mu) \\
9 \varepsilon z^{n}\end{array}$} \\
\hline \multicolumn{4}{|c|}{$(6 x)$ ssen $100 ! 1 ! 40$} & & $(6 x)$ & ofes & & \\
\hline
\end{tabular}




\begin{tabular}{|c|c|c|c|c|c|c|c|c|}
\hline \multirow{2}{*}{$\begin{array}{l}U^{235} \\
\text { (ut\%) }\end{array}$} & \multicolumn{4}{|c|}{ Safe Mass $(\mathrm{kg})$} & \multicolumn{4}{|c|}{ Critical Hass $(\mathrm{kg})$} \\
\hline & $u^{235}$ & Uranium & $\mathrm{UF}_{6}$ & $\mathrm{UO}_{2} \mathrm{~F}_{2}$ & $u^{235}$ & Uranium & $U^{6}$ & $\mathrm{UO}_{2} \mathrm{~F}_{2}$ \\
\hline 20 & 0.480 & 2.400 & 3.552 & 3.107 & 1.021 & 5.105 & 7.556 & 6.610 \\
\hline 21 & 0.475 & 2.260 & 3.345 & 2.926 & 1.009 & 4.803 & 7.109 & 6.219 \\
\hline 22 & 0.470 & 2.135 & 3.160 & 2.765 & 0.997 & 4.533 & 6.710 & 5.870 \\
\hline 23 & 0.465 & 2.023 & 2.995 & 2.620 & 0.987 & 4.291 & 6.352 & 5.556 \\
\hline 24 & 0.461 & 1.922 & 2.845 & 2.489 & 0.977 & 4.072 & 6.028 & 5.273 \\
\hline 25 & 0.457 & 1.830 & 2.708 & 2.369 & 0.968 & 3.873 & 5.734 & 5.016 \\
\hline 26 & 0.454 & 1.745 & 2.584 & 2.260 & 0.960 & 3.692 & 5.466 & 4.782 \\
\hline 27 & 0.450 & 1.667 & 2.469 & 2.159 & 0.952 & 3.527 & 5.222 & 4.568 \\
\hline 28 & 0.447 & 1.596 & 2.362 & 2.066 & 0.945 & 3.375 & 4.997 & 4.371 \\
\hline 29 & 0.443 & 1.529 & 2.264 & 1.980 & 0.938 & 3.235 & 4.790 & 4.190 \\
\hline 30 & 0.440 & 1.467 & 2.172 & 1.900 & 0.932 & 3.106 & 4.599 & 4.023 \\
\hline 31 & 0.437 & 1.409 & 2.086 & 1.824 & 0.926 & 2.986 & 4.422 & 3.868 \\
\hline 32 & 0.433 & 1.354 & 2.005 & 1.754 & 0.920 & 2.875 & 4.257 & 3.724 \\
\hline 33 & 0.430 & 1.303 & 1.930 & 1.688 & 0.915 & 2.772 & 4.104 & 3.590 \\
\hline 34 & 0.427 & 1.256 & 1.859 & 1.626 & 0.909 & 2.675 & 3.961 & 3.465 \\
\hline 35 & 0.424 & 1.211 & 1.793 & 1.568 & 0.905 & 2.585 & 3.828 & 3.348 \\
\hline 36 & 0.421 & 1.169 & 1.731 & 1.514 & 0.900 & 2.500 & 3.702 & 3.238 \\
\hline 37 & 0.418 & 1.130 & 1.673 & 1.463 & 0.896 & 2.420 & 3.585 & 3.135 \\
\hline 38 & 0.415 & 1.093 & 1.618 & 1.415 & 0.891 & 2.345 & 3.474 & 3.038 \\
\hline 39 & 0.413 & 1.058 & 1.567 & 1.370 & 0.887 & 2.275 & 3.370 & 2.947 \\
\hline 40 & 0.410 & 1.025 & 1.518 & 1.328 & 0.883 & 2.208 & 3.271 & 2.861 \\
\hline 41 & 0.408 & 0.994 & 1.473 & 1.288 & 0.880 & 2.146 & 3.178 & 2.780 \\
\hline 42 & 0.405 & 0.965 & 1.430 & 1.250 & 0.876 & 2.086 & 3.090 & 2.703 \\
\hline 43 & 0.403 & 0.938 & 1.389 & 1.215 & 0.873 & 2.030 & 3.007 & 2.630 \\
\hline 44 & 0.401 & 0.912 & 1.350 & 1.181 & 0.869 & 1.976 & 2.927 & 2.560 \\
\hline 45 & 0.399 & 0.887 & 1.314 & 1.149 & 0.866 & 1.925 & 2.852 & 2.494 \\
\hline 46 & 0.397 & 0.863 & 1.279 & 1.119 & 0.863 & 1.877 & 2.780 & 2.431 \\
\hline 47 & 0.395 & 0.841 & 1.246 & 1.090 & 0.860 & 1.830 & 2.712 & 2.372 \\
\hline 48 & 0.393 & 0.820 & 1.215 & 1.062 & 0.857 & 1.786 & 2.647 & 2.315 \\
\hline 49 & 0.392 & 0.799 & 1.185 & 1.036 & 0.855 & 1.744 & 2.585 & 2.260 \\
\hline 50 & 0.390 & 0.780 & 1.156 & 1.011 & 0.852 & 1.704 & 2.525 & 2.208 \\
\hline 51 & 0.388 & 0.761 & 1.128 & 0.987 & 0.849 & 1.666 & 2.468 & 2.158 \\
\hline 52 & 0.387 & 0.744 & 1.102 & 0.964 & 0.847 & 1.629 & 2.414 & 2.111 \\
\hline 53 & 0.385 & 0.727 & 1.077 & 0.942 & 0.845 & 1.593 & 2.362 & 2.065 \\
\hline
\end{tabular}

Final Report

June 1994
Minimum Critical Masses for Uranium at the Portsmouth Gaseous Diffusion Plant
Page 16 


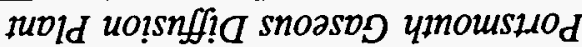

$\angle I \partial 8 v_{d}$

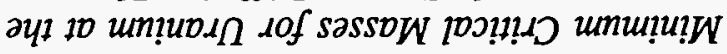

juodəy [Bu!H

\begin{tabular}{|c|c|c|c|c|c|c|c|c|}
\hline $9 \angle 1 \cdot 1$ & $97 E^{\circ} 1$ & $206^{\circ} 0$ & $68 L^{\circ} 0$ & $925^{\circ} 0$ & $109^{\circ} 0$ & $507^{\circ} 0$ & $\varepsilon S \Sigma^{\circ} 0$ & 28 \\
\hline $261^{\circ}$ & $79 \varepsilon^{\circ} L$ & $616^{\circ} 0$ & $06 L^{\circ} 0$ & 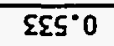 & $609^{\circ} 0$ & $0 เ 7^{\circ} 0$ & ESE.0 & 98 \\
\hline $802^{\circ} l$ & $285^{\circ} l$ & $1 \varepsilon 6^{\circ} 0$ & $162^{\circ} 0$ & $075^{\circ} 0$ & $\angle 19^{\circ} 0$ & $914^{\circ} 0$ & $7 S \varepsilon^{\circ} 0$ & $\$ 8$ \\
\hline $722^{\circ} b$ & $007^{\circ} 1$ & $776^{\circ} 0$ & $\varepsilon 6 L^{\circ} 0$ & $\angle 7 S^{\circ} 0$ & $929^{\circ} 0$ & $227^{\circ} 0$ & $755^{\circ} 0$ & 78 \\
\hline เฤट・| & $617^{\circ} 1$ & $956^{\circ} 0$ & $76 L^{\circ} 0$ & $755^{\circ} 0$ & $7 \varepsilon 9^{\circ} 0$ & $\angle 27^{\circ} 0$ & SSE.0 & 58 \\
\hline $852^{\circ} 1$ & $6 \Sigma^{\circ} 7^{\circ} \mathrm{L}$ & $0 \angle 6^{\circ} 0$ & $56 L^{\circ} 0$ & $295^{\circ} 0$ & $\varepsilon \neq 9^{\circ} 0$ & 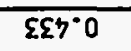 & SSE. 0 & 28 \\
\hline $\sin 1$ & $657^{\circ} 1$ & $286^{\circ} 0$ & $96 L^{\circ} 0$ & $0 \angle 5^{\circ} 0$ & $259^{\circ} 0$ & $6 \Sigma \eta^{\circ} 0$ & $9 S \varepsilon^{\circ} 0$ & 18 \\
\hline$\varepsilon 62^{\circ} l$ & $6 \angle 7^{*} L$ & $266^{\circ} 0$ & $86 L^{\circ} 0$ & $8 \angle 5^{\circ} 0$ & $199^{\circ} 0$ & $944^{\circ} 0$ & $95 \varepsilon^{\circ} 0$ & 08 \\
\hline ZIE'l & $005^{\circ} 1$ & $110^{\circ} !$ & $662^{\circ} 0$ & $985^{\circ} 0$ & $1 \angle 9^{\circ} 0$ & $257^{\circ} 0$ & $\angle S E^{\circ} 0$ & $6 L$ \\
\hline $\mid \varepsilon \varepsilon^{\circ} !$ & $225^{\circ} 1$ & $920^{\circ} \mathrm{l}$ & $008^{\circ} 0$ & $565^{\circ} 0$ & $189^{\circ} 0$ & $657^{\circ} 0$ & $85 \varepsilon^{\circ} 0$ & 82 \\
\hline $05 E^{\circ} !$ & $575^{\circ} l$ & $b 70^{\circ} l$ & $208^{\circ} 0$ & $709^{\circ} 0$ & $169^{\circ} 0$ & $994^{\circ} 0$ & $8 S E^{\circ} 0$ & $L$ \\
\hline $0 \angle \varepsilon^{\circ} l$ & $295^{\circ} !$ & $\angle 50^{\circ} 1$ & $\varepsilon 08^{\circ} 0$ & $\varepsilon 19^{\circ} 0$ & $10 L^{\circ} 0$ & 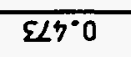 & $65 \varepsilon^{\circ} 0$ & 94 \\
\hline $16 \varepsilon^{\circ} l$ & $665^{\circ} l$ & $\varepsilon \angle 0^{\circ} l$ & $708^{\circ} 0$ & $229^{\circ} 0$ & $2 L^{\circ} 0$ & $087^{\circ} 0$ & $09 \varepsilon^{\circ} 0$ & SLl \\
\hline $217^{\circ} b$ & $519^{\circ} b$ & $680^{\circ} \mathrm{L}$ & $908^{\circ} 0$ & $2 \varepsilon 9^{\circ} 0$ & $\varepsilon 2 L^{\circ} 0$ & $887^{\circ} 0$ & $19 \varepsilon^{\circ} 0$ & 72 \\
\hline $7 \varepsilon 7^{\circ} l$ & $049^{\circ} 1$ & $901^{\circ} 1$ & $208^{\circ} 0$ & $2+9^{\circ} 0$ & $S E L^{*} 0$ & $567^{\circ} 0$ & $29 \varepsilon^{\circ} 0$ & $\varepsilon$ \\
\hline$\left\langle s^{\circ} l\right.$ & $999^{\circ} 1$ & $\varepsilon 2 l^{-} l$ & $608^{\circ} 0$ & $\Sigma 59^{\circ} 0$ & $\angle 7 L^{\circ} 0$ & $\cos ^{\circ} 0$ & $29 \varepsilon^{\circ} 0$ & 22 \\
\hline $087^{\circ} !$ & $\varepsilon 69^{\circ} L$ & 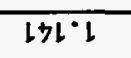 & $018^{\circ} 0$ & $799^{\circ} 0$ & $6 S L^{\circ} 0$ & $215^{\circ} 0$ & $\varepsilon 9 \varepsilon^{\circ} 0$ & $\overline{L L}$ \\
\hline $705^{\circ} 1$ & $02 L^{\circ} 1$ & $091^{\circ} \mathrm{l}$ & $218^{\circ} 0$ & $S \angle 9^{\circ} 0$ & 22100 & $025^{\circ} 0$ & $79 \varepsilon^{\circ} 0$ & $0 L$ \\
\hline $625^{\circ} 1$ & $87 L^{\circ} !$ & $6 \angle L^{\circ} l$ & $\varepsilon 18^{\circ} 0$ & $989^{\circ} 0$ & $S B L^{\circ} 0$ & $625^{\circ} 0$ & $59 \varepsilon^{\circ} 0$ & 69 \\
\hline $755^{\circ} 1$ & $8 L L^{\circ} l$ & $666^{\circ} \mathrm{L}$ & $518^{\circ} 0$ & $869^{\circ} 0$ & $662^{\circ} 0$ & $8 E 5^{\circ} 0$ & $99 \varepsilon^{\circ} 0$ & 89 \\
\hline $185^{\circ} !$ & $808^{\circ} l$ & $612^{*} l$ & $\angle 18^{\circ} 0$ & $G L^{\circ} 0$ & $\varepsilon 18^{\circ} 0$ & $875^{\circ} 0$ & $\angle 9 \varepsilon^{\circ} 0$ & $\angle 9$ \\
\hline $809^{\circ} 1$ & $6 \varepsilon 8^{\circ} L$ & $072^{\circ} L$ & $818^{\circ} 0$ & $\varepsilon 2 L^{\circ} 0$ & $228^{\circ} 0$ & $855^{\circ} 0$ & $89 \varepsilon^{\circ} 0$ & 99 \\
\hline $959^{\circ} 1$ & $1 \angle 8^{\circ} !$ & $292^{\circ} 1$ & $028^{\circ} 0$ & $\angle L^{\circ} 0$ & $278^{\circ} 0$ & $895^{\circ} 0$ & $69 \varepsilon^{\circ} 0$ & 59 \\
\hline$\$ 99^{\circ} \mathrm{L}$ & $706^{\circ} !$ & $782^{\circ} !$ & $228^{\circ} 0$ & $O S L^{\circ} 0$ & $858^{\circ} 0$ & $6 \angle 5^{\circ} 0$ & $0 \angle \varepsilon^{\circ} 0$ & 49 \\
\hline $569^{\circ} 1$ & $6 \varepsilon 6^{\circ} 1$ & $\angle O \varepsilon^{\circ} b$ & $728^{\circ} 0$ & $59 L^{\circ} 0$ & $728^{\circ} 0$ & $065^{\circ} 0$ & $2 \angle \varepsilon^{\circ} 0$ & $\varepsilon 9$ \\
\hline $922^{\circ} 1$ & $7 \angle 6^{\circ} l$ & $2 \varepsilon \varepsilon^{\circ} L$ & $928^{\circ} 0$ & $6 \angle L^{\circ} 0$ & $168^{\circ} 0$ & $109^{\circ} 0$ & $\varepsilon \varepsilon^{*} 0$ & 29 \\
\hline $8 S L^{\circ} 1$ & $110^{\circ} 2$ & $95 \varepsilon^{\circ} 1$ & $\angle 28^{\circ} 0$ & $S 6 L^{\circ} 0$ & $606^{\circ} 0$ & $\varepsilon 19^{\circ} 0$ & $\eta \angle \varepsilon^{\circ} 0$ & 19 \\
\hline $26 L^{\circ} 1$ & $670^{\circ} 2$ & $28 \varepsilon^{\circ} \mathrm{L}$ & $628^{\circ} 0$ & $118^{\circ} 0$ & $226^{\circ} 0$ & $529^{\circ} 0$ & $S \angle E^{\circ} 0$ & 09 \\
\hline $228^{\circ} L$ & $680^{\circ} 2$ & $607 \%$ & $1 \xi 8^{\circ} 0$ & $\angle 28^{\circ} 0$ & $976^{\circ} 0$ & $8 \varepsilon 9^{\circ} 0$ & $\angle \varepsilon^{\circ} 0$ & 65 \\
\hline$\varepsilon 98^{\circ} !$ & $0 \varepsilon L^{\circ} 2$ & $\angle \varepsilon \nabla^{\prime} l$ & $\varepsilon \varepsilon 8^{\circ} 0$ & $748^{\circ} 0$ & $996^{\circ} 0$ & $159^{\circ} 0$ & $8 \angle \varepsilon^{\circ} 0$ & 85 \\
\hline $006^{\circ} \mathrm{L}$ & $\varepsilon L l^{*} Z$ & $997^{\circ} 1$ & $9 \varepsilon 8^{\circ} 0$ & $298^{\circ} 0$ & $986^{\circ} 0$ & $599^{\circ} 0$ & $6 \angle \varepsilon^{\circ} 0$ & $\angle 5$ \\
\hline $6 \varepsilon 6^{\circ} \mathrm{l}$ & $\angle 12^{\circ} 2$ & $967^{\circ} 1$ & $8 \varepsilon 8^{\circ} 0$ & $188^{\circ} 0$ & $800^{\circ} \mathrm{L}$ & $089^{\circ} 0$ & $18 \varepsilon^{\circ} 0$ & 95 \\
\hline $626^{\circ} !$ & $592^{-2}$ & $225^{\circ} 1$ & $058^{\circ} 0$ & $006^{\circ} 0$ & $0 \varepsilon 0^{\circ} b$ & $569^{\circ} 0$ & $28 \varepsilon^{\circ} 0$ & 55 \\
\hline $120^{\circ} 2$ & ZIE"2 & $095^{\circ} \mathrm{L}$ & $2+8^{\circ} 0$ & $126^{\circ} 0$ & $\varepsilon S 0^{\circ} \mathrm{L}$ & $O L L^{\circ} O$ & $78 \varepsilon^{\circ} 0$ & 75 \\
\hline $\mathrm{z}^{2} \mathrm{on}$ & $\overline{9} \mathrm{gn}$ & un!̨uedn & $\operatorname{sez} n$ & $t^{2}$ on & ${ }^{9} \mathrm{~A} \cap$ & unţuesn & $s \in z^{n}$ & \multirow{2}{*}{$\begin{array}{l}(\%+4) \\
9 \varepsilon t^{n}\end{array}$} \\
\hline \multicolumn{4}{|c|}{$(6 y) \operatorname{ssen} 1 e 0 ! 7 ! 13$} & & (E) & 42 & & \\
\hline
\end{tabular}


POEF-SH-2O

\begin{tabular}{|c|c|c|c|c|c|c|c|c|}
\hline \multirow{2}{*}{$\begin{array}{l}U^{235} \\
(w t \%)\end{array}$} & \multicolumn{4}{|c|}{ Safe Mass (kg) } & \multicolumn{4}{|c|}{ Critical Mass (kg) } \\
\hline & $\mathrm{U}^{235}$ & Uranium & $u F_{6}$ & $\mathrm{UO}_{2} \mathrm{~F}_{2}$ & $\mathrm{u}^{235}$ & Uranium & $\mathrm{UF}_{6}$ & $\mathrm{UO}_{2} \mathrm{~F}_{2}$ \\
\hline 88 & 0.352 & 0.400 & 0.594 & 0.519 & 0.788 & 0.895 & 1.329 & 1.161 \\
\hline 89 & 0.352 & 0.395 & 0.586 & 0.513 & 0.787 & 0.884 & 1.312 & 1.147 \\
\hline 90 & 0.351 & 0.390 & 0.579 & 0.506 & 0.786 & 0.873 & 1.296 & 1.132 \\
\hline 91 & 0.351 & 0.385 & 0.572 & 0.500 & 0.784 & 0.862 & 1.280 & 1.118 \\
\hline 92 & 0.350 & 0.381 & 0.565 & 0.494 & 0.783 & 0.851 & 1.264 & 1.105 \\
\hline 93 & 0.350 & 0.376 & 0.559 & 0.488 & 0.782 & 0.841 & 1.249 & 1.091 \\
\hline 94 & 0.350 & 0.372 & 0.553 & 0.483 & 0.781 & 0.831 & 1.234 & 1.078 \\
\hline 95 & 0.350 & 0.368 & 0.547 & 0.478 & 0.780 & 0.821 & 1.219 & 1.066 \\
\hline 96 & 0.350 & 0.365 & 0.541 & 0.473 & 0.779 & 0.812 & 1.205 & 1.053 \\
\hline 97 & 0.350 & 0.361 & 0.536 & 0.468 & 0.778 & 0.802 & 1.191 & 1.041 \\
\hline 98 & 0.350 & 0.357 & 0.530 & 0.463 & 0.777 & 0.793 & 1.177 & 1.029 \\
\hline 99 & 0.350 & 0.354 & 0.525 & 0.459 & 0.776 & 0.784 & 1.164 & 1.017 \\
\hline 100 & 0.350 & 0.350 & 0.520 & 0.454 & 0.775 & 0.775 & 1.151 & 1.006 \\
\hline
\end{tabular}

